\title{
Namenregister Nr. 14
}

Ein vollständiger oder gekürzter Abdruck der Referate ist ohne Genehmigung der Redaktion und des Verlages verboten!

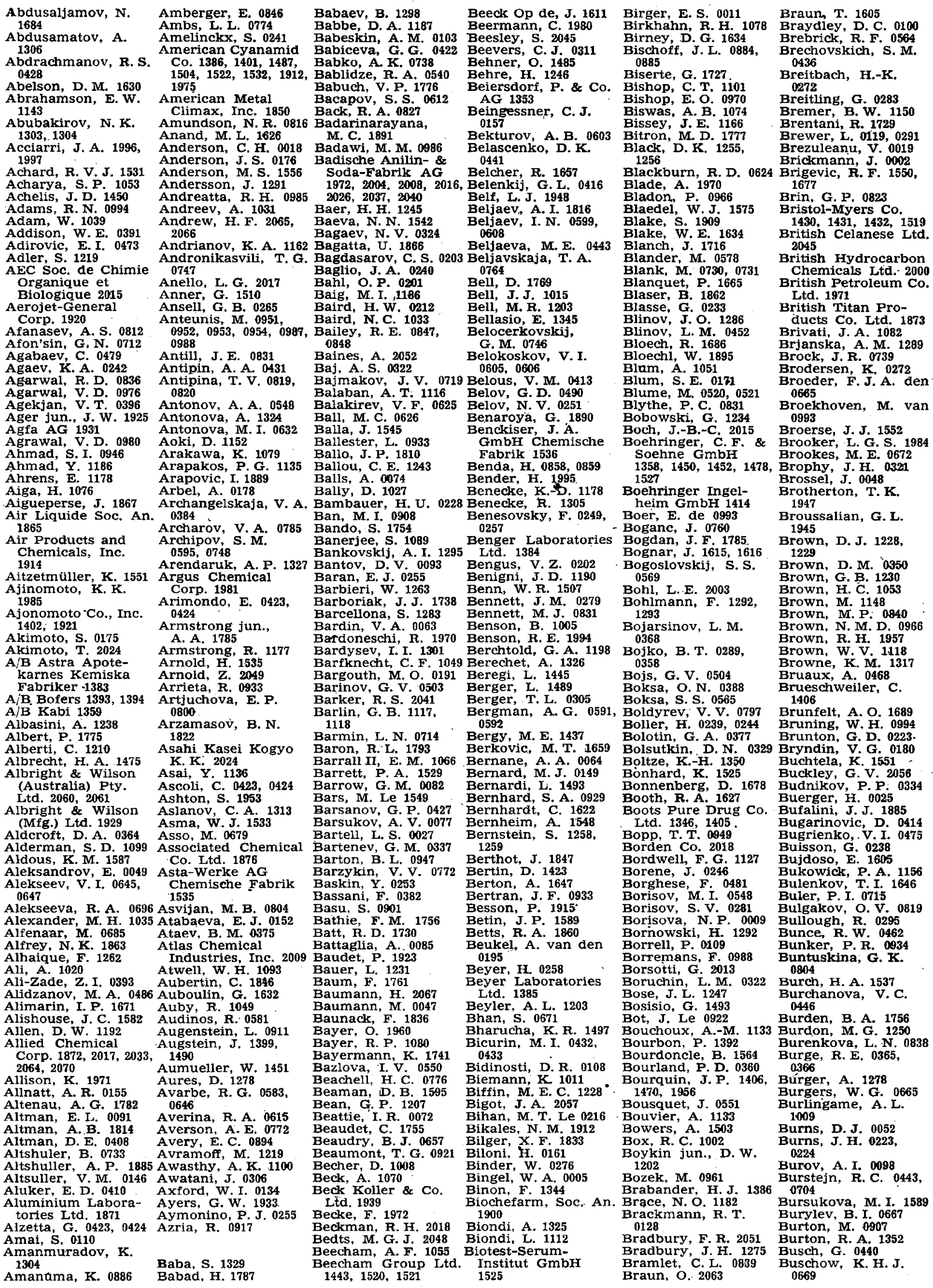




Buslaev, J. A. 0069,
0076
Buss AG 1859
Busse-Macukas,
V. B. 0660
Buttrey, D. E. 0038
Butler, A. K. 1145
Butler, K. H. 0390
Buurman, R. den
0195
Buxton, M. W. 1943,
1948
Buzano, C. 0105
Bydin, J. F. 0122
Bykova, E. V. 0782
Bykova, I. N. 0596
Byrn, S. R. 1147
Bystrov, L. M. 0357

Cabana, A. 0084
Cable, J. W. 0523
Ceskoslovenska akademie ved 2049 Chacaturjan, A. G. 0338

Chaffey, G. H. 0831 Chain, V.S. 077 Chaiwasie, S. 0247
Chakimdzanov, S 1306 Chakrabarti, S. K. 0925, 0927 Chakrabartty, S, K. Chaldeev, G. V. 018 Chalmers, B. 016 Chan, S. C. 086 Chan, S. I. 0967 Chan, T. H. 1277 Chandrasekhar, G. V.

Chang, C. M. 1738

Cacanidze, G. D. 1619 Channing, J. E. 0940 1003
Cagarejsvili, D. S.

Caglioti, L. 1263 Caglioti, L. 1263 Cagunava, J. W. 0285 Caillet, M. 1673 Cainelli, G. 1263 Cajka, M. 0049 Cajka, M. P. 0091 Cakadze, D. S. 0539 Calcagno, B. 184. Caldini, O. 1179 California Research Corp. 2010

Call, D. R. 1123 Camarn, P. 0177 de 0219

Camerino, B. 1493 Campaigne, E. E. 1 Campazzi, G. 1893 2052 Charachorin, F. F.

0056, 0057, 0058, 0059,
005aritonov, J. J. 0060, 0061, 0076

2 Charkac, J. I. 0814 Charney, E. 0929 13

Charus, G. I. 0455

Chatterjee, A. K. 0741 Chatterjee, G. 0124

Chawla, G. R. 1170

Chawla, I. D. 1800

Chayen, R. 1748

Chejfec, V. I. 1776

Phosphates Ltd. 1413

Chemische Fabrik Kalk GmbH 1839,

1946
Chemische Fabrik Pfersee GmbH 191 Chemische Fabrik Schweizerhall 139 Witten GmbH 202 Chen, E. 1075

Campbell, D. 0992 Chen, E. 1075

Campbell, I. A. 0.515 1496, 1497

Chen, Jo-Yun 1697 1235

Canterford J. H, 0028 Cheng, C. S. 2033 Cantor, C. R. 0891 Chesswas, M. 0173

Cantu, A. A. 0014 Caproaso, A. J. 1868 (171 J. A. A. 0377 Carlson, E. J. 1872 Carlson, G. A. 1896 . Chevaleyre, J. 1133 Co. 1938

Cheymol, G. 1328 Cheymol, J. 1328 Chhabra, V. K. 1170 Chicotka, R. J. 0171 Childs, B. G. 0331 Carmichaei, R. S. 0534 Chilton, W. S. 114 Carnevale, E. H. 0117 Chinoin Gyogy'szere Caro, A. N. 1227 Vegyeszeti Carpeni, G. 0678, 0679 Carpenter, M. Carlston, C. E. 0128 Carter, J. G. 0895 Carter, w. B. 1996 Carunchio, V. 0789 Casaretti, L. 132 Case, F. H. 1197 Cassada, E. 1271 Cassady, J. M. 100 Cassidy, R. M. 1680 Castagnou, R. 166 Catino, S. C. 1982 Cavalla, J. F. 1379 Cave, G. C. B. 1067 Cebotarev, N. T. 00 Cechmataeva, G. D. ching

Cechov, E. E. 1881

Cefola, M. 0229

Celeda, J. 0766

Cellarius, R. A. 1012 Cepeleva, I. V. 0435 Cercon, L. A. 1979 Cerkasov, E. M. 0035 Cernik. I. A. 0478 Cernjaev, L. A. 1822 Cernjak, G. E. 0465 Cernjak, V. G. 0409 Cernjak, V. V. 1586 Cernjavskij, B. G. Termekek R. T. 1517 Chinson, R. G w Chlebarova, E. I. 1286 Chochlova, J. M. Cholodova, G. V. 1288 Cholzaev, O. F. 0425 Chompff, A. J. 126
Chong, D. M. 0765 Christensen, C. R.

Christian, G. D. 1700 Christophorou, L. G.

Christy, M. E. 1360 Chu, W. K. 0360
Chudgar, A. J. 1785 Chudgar, A. J. ${ }^{1785}$ Chusidman, M. B. 0430

Chutjian, A. 0119 C812 Chvorostenko, A. S.

Ciba AG 1454, 1464, Ciba Ltd. 1347. 1390, $1396,1412,1433,1439$ Ciba Soc. An. 1380 ,

1848 . 18 . N. Cicisvili, G. V. 0747 Cidilkovskij, I. M. 0455

Cernov, S. A. 0410
Cipera, J. 1821 Cirkovic, S. 1889 Claeson, C. G. 1393 Clark, J. K. 1043 Clark, W. 0381 Claude, J.-R. 1740


1014 . 1873 Cleaver, D. 1873 Clementi, E. 1036 , 1037,1038
Clementi, H. 1036 Clementi, H. 1036 Coad, J. R. 1106 Coates, H. 2060 Coates, W. H. 1876 Coats, J. H. 1437 Cochet-Muchy, B. 184 Cochran, L. W. 183 Cockayne, B. 0173 Cohen M 0013 Cohen, V. I. 1284 Coleman, L. B. 0278 Colleter, J. C. 105 ? 1058

Collins, J. D. 1929 Colton, R. 0028 Combes, M. 1548 Comeaux, A. R. 0128 Commins, B. T. 1654 Commissariat a
l'Energie Atomique 1846, 1847 Atomique Comp. Francaise de Raffinage 1998 Compernolle, F. 0951 Comyns, A. E. 1873 Cone, C. 1011 Conkerton, E. J. 170 Conlon, M. 1759

Conrad, H. 0186 Conrow, R. B. 1258, 125

Constantinescu, E.$$
1326
$$

Conti, J, J, 1069 Continental Oil Co. 1996, 1997

Cook, A. F. 1251 Cook, R. L. 0353 Cook, S. S. 1952 Cook, W. S. 1782 Cooper, L. E. 2007 Cooper, R. L. 0864 Cornforth, J. W. 1322 Cornforth, R. H

1322

Corre, F. 1740

Cotton, F. A. 0263

Cowan, D. O. 0278

Cox, B. 0810

Crawford, 1198 Crescenzi, G. S. 128

Crocket, J. H. 1683

Croizet, J. 1665

Cross jun., R. J. 0111

Crousier, J.-P. 0793

Crow, D. R. 0674
Cruickshank, D. W. J. Cruicis

Cruse, R. 2047 Cuchlancev, V. G.


Cucmarev, S. K. 081 Cudinov, A.S. 0687 Cufarov, G. I. 0625
Cugunnikova, R. V

Cukor, P. 1670

Cukerblat, B. S. 0405

Culik, K. 1434, 1435

Curagulov, B. R. 056

Curikova, G. A.
Czech, F. P. 1788

\section{Diprose, G, L. A}

Dirheimer, G. 172

Disteldorf, J. 1961

Dagnall, R. M. 1587 Dagra, N. V. 1954 K. K. 1440 Dairova, A. M. 0664 Dalev, D. 1334 Damany, N. 0042 Dancewicz, D. 1139 Dancewis, J. 1746 Dane, L. M. 0711 Daniel, J. S. 0314 Daniels, W. B. 0524 Davies, G. R. 0262 Davis, D. F. 0683 Davis, D. R. 1036 Davis, J. E. 1565 Davis jun., J. C. 0971

Davison, B. K. 1441 Davydova, S. L. 0823 Daweke, H. 1737 Dets, P. C. 0252 Debuigne, J. 020 Decamps, E. 0395 Decomps, B. 004 Dehmelt, H. G. 0050 ehydag Deutsche Dejneka, G. F. 0604 Delavignette, P. 024 Ltd. 1398

Delorme, P. 0938 Delzenne, A. 1887 Dembovskij, S. A Denga, E. M. 0475 Denisov, E. P. 1592 Denisov, F. V. 1810 Denschlag, H. O. Deputy Minister of the Rumanian

Danilchenko, V. E. Dobrihalova, L. 0933 Efimceva, O. P. 1279 Danilova, N. 0656 1332 Dolskij, N. N. 0170 J. V. 1614 Darby jun., J. B. 0275, 1617 Efros, A. L. D489 Dobrusina, G. D. 0764 Egan, B. Z. 1637 Darvojd, 'T. I. 0379 Dodd, R. A. 0194 ${ }^{2}$ Egorenko, G. A. 0629 Das, T.R. 0547 Doehnert, H. 1307 Egorockin, A. N. 009 Datta, P. 0082 Dogadkin, N. N. 1671 Egorov, V. N. 0035 Davies, D. B. 1061 Doherty, J. 1793 Eguchi, S. 1985 Davies, W. 1215 Donato, E. 0481 1301 Ehmann, L. 1510 Davidovic, R. L. 0069 Donnay, G. P278 1301 Ehrhart, G. 1444 14 Davies, D. W. 0004 Donovan, J. J. 1914 
Fasman, A. B. 0702 Faubl, $H$. B. Faust, G. 1955 Fawkes, J. 1625 Fayez, M. B. E. 0986 Fayolle, J. C. 1548 Fearon, F. W. G. 0854 Fechretainov, F. A.

edorenko, V. D. 060 edorov, G. B. 0662 Fedoseeva, S. N. 1822 Fedotova, T. D. 009 Feinberg, M. J. 0024 Feit, I. N. 1122 Feldgun, L, I 0196 Feldman, C. 0287 Fenn, $R$, $F 247$ Fennessey, J. P. 0248 Fernholz, H. 2019 Eersht, A. R. 1104 , 1105

Fesenko, E. G. 0499 Fessenden, J. S. 1164 Fessenden, R. J. 1164 Eickentscher, K. Fielding, B. C. 1840 Fielder, M. L. 0440 Fiedler, W. 1955 Figeys. H. P. 0964 Filip, B. 0341
Filipenko, O. S. 025 Filippin, V. A. 0545 Filler, R. 1154 Filonenko, N. E.
Findeis, A. F. 0910 Fink, G. 0150 Finley, K. T. 1123 Fischer, D. 1628 Fischer, E. 1411 Fischer, E. O. 0864 Fishbein, L. 1625 Fisher, F. F. 0683 Fishekis, J. 1230 Fissekis, J. 1230 Fite, W. L. 0128 Fitzgerald, D. J. 0450 Fitzmaurice, C. 136 Fivejskij, E. V. 0645 Flamming, R. 0964 F160

Flerova, S. A. 0503

Fletcher, E. A. 0774 Flowers, H. M. 124 Flurry jun., L. 1015 FMC Corp. 1919 Foersterling, H. D. 1010 Foerstner, G. 0351 Foerstner, G. 0351 Foner, A. 1219 Fontanella, L. 1409, 1410

Forbes, D. H. 0165 Forostjan, J. N. 1302 out . C. 1220 Fox, M Frackowlak, D. 0930 Fraenkel, G. K. 098 Frame, R. R. 1127 Franck-Neumann, M 1212

Frank1, D. R. 0448 Franklin, G. M. 1825 Franzen, H. F, 0234 Fraser, R. R. 1046 Frawley, J. J. 0164 Frazer, B. C. 0524 Frei, K. 1090 Freiberg, W. 1086 1087

Freifelder, M. 0972 French, R. D. 0444

Freund, H. G. 1092 Freund, $T$. 0836 Freundlich, w, 024 Freymann, R. 0078 Freyschlag, H. 2016 Freytag, H. 1936 Freytag, K.-H. 2069 Friary, R. J. 1160 Fricke, H. 0828 Frid, A. S. 0613 Fridovich, I. 1159 Frieberg, V. J. ${ }^{\theta 498}$ Friebolin, H. Fried, J. 1511 1455
Friedmann, G. 1272, Gibbon, C. F. 0296, Fringuelli, F. 1254 Frisch, E. E. 2002 Fritzsch, K. 0538 Frodyma, M. M. 1726 From, Fruhstorfer, W. 1467 ry, A. J. 1193 Fryer, R. I. 1489 Fujibar, R. I. 148 0403 Fulii, M. 0958 Fujimoto, H. 1311 Fujimoto, M. 0990 Fujimoto, Y. 1440 Fujita, A, 1440 Fukui, S. 1690 Fuller jun., E. L. 075 Fuller, F. D. 1758 Furuyama, S. 1128 Futrell, J. H. 1138 y fe, W. S. 0884

Gabriel, A. H. 0137 Gabuda, S. P. 009 Gadret, M. 1057, 1058 Gaeumann, A. 0633 Gagliardi, D. D. 1931 Gagnaux $P$. 1406 Gaia, F. 1859 Gajducenja, V. F 0320

Galcenko, G. L. 0553 Galcenko, I. E. 1817 Galimov, D. G. 0438 Galkin, A. A. 0540 Galkin, G. N. 0452 Galkin, V. V. 0461 Gallinella, E. 201 Gamboni, G. 1956 Ganeev, $G$, 0267

Ganiev, A. G. 1684

Gar, T. K. 0098

Garbutt, G. B. 0990

Gard. E. 1116

Garg, S. K. 0916

Garifjanov, N. S. 0428 Garrett, C. 179

Garrett, E. R. 1716

Gashurov, G. 0240

Gavriljacenko, V. G 0499

Gawargious, Y. A. 1687

\section{R. L. 1189}

Gebauer, P. A. 1099

Gede, M. F. I58. Geigy, J. R. AG 1371, $1372,1408,1415$

Geigy Ltd. 1909 Geisler, H. 0685 Gejo, T. 1827 Gelan, J. 0988 General Aniline \& Film Corp. 1982 $2012,2029,2034$ 2012, 2029, 2034
General Electric Co.

Genkin, V. N. 0454

George, F. C. K. 1745

George, M. V. 0080 Georgieva, I. C. 128 Gerard, J. 1 15B2 Gerasimov, J. M. 0170

Gergel, V. A. 0451 Gergovich, H. J. 202 2022. Grant, N. j. 0271 Germerdonk, R. 1824 Grass, F. 1551 . Gerok, W. 174 Gibs, G. J. 1224 Gibson, N. A. 1177 Giessen, B. C. 0271 Gilev, A. P. 1323 Gilfanov, F. Z. 0412 Gillap, W. R. 0742 Gillespie, R. J. 0977 , Gillieson, A. H. 1590 Cillingham, J. T. 1807 Gilman, H. 0854 Gilson, T. 0072 Ginderow, D. 0197 Giovandetti, 0284 Gittins, A. 0192, 0303 Giuli, G. De 1254

Gjunner, E. A. 0617 Glaxo Laboratories Ltd. 1495 Glazov, V. M. 0572
Glazunov. P. J. 0367 Gleiter, H. 0179

Gilemser, O. 0258

Glistenko, N. I. 0636 Gluchareva, N. A.

1608

Gluzinski, P. 0939、

Gmuender, J. 1465

Gocalieva, E. P. 0721

Godfrey, M. I030

Godina, N. A. 0623

1513

Goffredo, O. 1493

Goginjan, E. A. 1878

Golcman, B. M. 0

Goldman, A.

Goldschmidt, T. AG

Goldsmith, R. L. 039 Goldstein, C. 1270
Goldwhite, H. 1166 GoldWhite, H. 1166 Golger, J. J. 0754

GolikoY, V. M. 0349

Golodec, G. I. 0818

Goncarov, D. N. 1554

Gonnert, R. 2032

Goodall, R. R. 1624

Goud6

Goot, A. S. van der

Gorbacev, S. K. 0142

Gorbatyj, N. A. 0446,

Gordon, M. 1018

Gorin, P. A. J. 1052 Haas, T. E. 0024

Gorjunova, N. P. 0579 Haaver, E. 2008

Gorjusko, A. G. 0421 Hackman III, E. E.

0688

Gorogeckaja, L. I.

028

Goroscenko, J. G. 0606

Gosnell, C. M. 1604

Gosselin, A. 0020

Goto, K. 2024 19

Gough, T. E. 1000 1048

Gould, J. H. 1697

Govil, G. 0962

Grabhofer, H. 1931

Grace, W. R \&

1356, 1863 . 1327

Graceva, E. A. 132

0466 Green J. H. S. 0073

Gersmann, H. R. 2039 Greene, C. R. 2005

Geschke, D. 0744

Gesser, H. D. 09

Getoff, N. 0829
Gharpurey, M. K.

1074

Greenwood, iN. N.

Greet, R. J. 1062

Greichus, Y. 1790

Grenda, V. J. 1183

Ghosh. S. 0960, 0861,

0862,0863

Giacomello, G. 1262

Giancotti, V. 0220

Gribulja, V. B. 064

Gricyna, V.V. ${ }^{0036}$

Gridasova, R. K.

Grieco, M. J. 0470

Giardina, M. C. 1262
Gibaldi, M. 0742

Gronlund, $H$. 1721

ronlund Thomsen

Grosh. J. 0541

OSS, E. F. 0396

ossmith, F. 1443

Grosvald, $G, 092$

Groth, U. 1741

Grover, A. R. 2043

Gruenklee, G. 1737

rum-Grzimajlo,

Grumbach, F. 1284

. 1081

ruver, R. M. 0330

Grigorescu, E. 1326 Harker, R. I. 0167

E. A. 0599 Harmer, G. L. M. 1405 1365, 1395, 1418, 1442

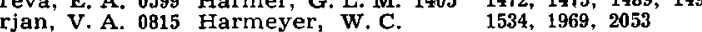



Grimison, A. 1039 Harmon, A. B. 1099

Grinberg, A. A. 0762, Harris, D. C. 0882

Harris, I. R. 0277

Grisin, V. P. 0158 Harris, N. H. 0353

Harrison, A. G. 1096

Hawley, M. D. 1569 


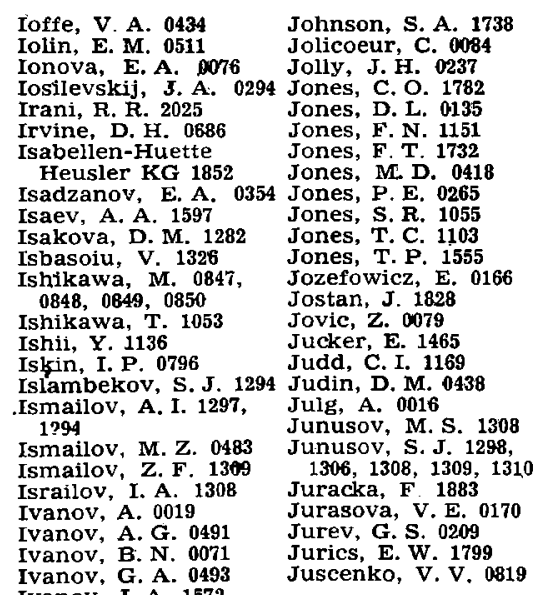

Ivanov, G. A. 0493

Ivanov, J. A. 1572

Ivanov, M. G. 1600
Ivanov, N. P. 1764

IVanov, O. S. 0310

.

Ivanovskij, G. F. 0346 Kacurin, G. A. 0480

Ivanovskij, M. N. 0570 Kadaner, E. S. 0658
Iverson, J. L. 1795

Iverson, J. L. $1795 \quad$ Kadenaci, B. M. 0635
Iwaki, H. 0584

Twaki, H. 0584 4. 1140

Izasa', 1004,1140

Jacenko, S. P. 0656
Jachkind, N. D. 0617

Jachkind, N. D. $\mathbf{0 6}$

Jacimirsi,
Jacin, 1806

Jacin, H. 1806
Jackson jun., W. J.

1963 Jun., W.

Jackwerth, E. 1789
Jacobs, P. W. $M$.

Jacobs, 0568

Jacobs, T. L. 1187

Jacobsen, G. 2019

Jacobsen, R. T. 1269 1793

Jacques, J. K. 1929

Jacques, J. K. 1999
Jaffe, P. M. 1869

Jaffe, P. L. 1265

Jakovlev, Y. V. 1671

Jakovleva, Z. S. 0412

Jambor, J. L. 0881,

Jambor

James, C. L. 1094

Jancevskaja, I. S. 04

Jancuk, A. F. 07.

Jankelevic, R. G.

Janke

Jankovskij, A. A.

0141

Janssen, L. J. J 0711

Janzen, E. G. 1093

Jarandina, V. N. 005

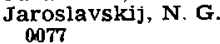

Jarysev, G. M. 0743

Jasinski, A. 0961

Jasko, A, O. 0749

Jasvili, T S. 0552

Jaul, E. 202

Jean-Louis, F. 0314

Jefferts, K. B. 0050

Jefís, P W 1239

Jehanno, G. 01

Jelen, K. 1612

Jelenic, I. 1667
Jenkins, P. W. 198

Jensen, H. P. 1794

Jensen, M. A 0970

Jesenak, V, 0341

Jesson, J. P. 0426
Jhon, Mu Shik 0541

Jobe, J D. 0116

Joffrin

Graffouillere, C.

Johannis, J. 2032

John, R. M. 0116
Johns, E. W. 1749

Johns, E. W. R. 1802

Johnson, D. A. 1519
Johnson, D. W. 0840

Johnson, D. W.
Johnson, F. 1146

Johnson, F. F. 1066

Johnson, M. D 110

Johnson, Q. 0254

1073

Kai, F. 0856

Kaiman, S. 1599

Kainz, G. 1694

Kaiser, D. G. 1340

Kajdanov, V I.

Kajgor

Kalberer-Ruesch,

M. E. 1524

Kaloev, N. I. 0597

Kalopissis, G. 1918

Kamada, H, 0974

Kamenceva, N. M.

0196

Kameneckaja, S. A

0834

Kametani, 'T. 1167 Kaminski, $F$ E.

Kanazawa, Y $\mathbf{Y 9 6 9}$

Kane, P O. 1573

Kanekar, C. R. 0962

Kapitonov, V. I. 0655

Kappelmeyer, R. 1

Kar38

Karapetjanc, M. C.

Karasik, E. A. 0460

Kareh, T. B. E. 090

Karelin, A. I. 0057,

$0058,0059,0060$

Karimdzanov, A. K

1294, 1297 .

Karjakin, A. V. 0422

1819

Karlov, N. V 0088

Karov, Z. G. 0780

Karpova, T. P. 0274

Karpovic, I. R. 1617
Kartasov, E. M. 0337

Kasehagen, L. 2009

Kasen, M. B. 0325

Kasina, N. I. 0595

Kasmaliev, B. 0140

$\begin{array}{ll}\text { Kasprzak, J } & 0729 \\ \text { Katchian, H. } & 1194\end{array}$

Katekar, G. F. 0984

Kates, M. 1277

Kato, A. 0876
Kato, H. 0958, 1208

Kato, T. 1218

Katritzky, A. R. 108

Katsuya, N 198

Katyl, R. H. N. 0431

Katz, L. 0265
Kawai, N. 0574

Kawakami, K. 0097 166

Kawaoka, K. 0898

Kawasaki, S. 0342

Kawashima, T. 1658

Kawazoe, Y. 1115

Kazarinov, R. F. 045

Kearns, D. R. 089

Kearvell, A. 0892
Keays, R. R. 1683

Kebrle, J. 1510

Keenan, J. F. E. 1909

Keese, H. 1753

Keh. A. S. 0318
Kekelidze, G. P. 0463

Kekelidze, N. P. 0463

Kelen, T. $0767,{ }^{0768}$
Keler, E. K. 0623

Keler, E. K. 0623

Keller, H. 1767
Kelley, P. W. 1758

Kelly, H. C. $\mathbf{1 6 6 0}^{160}$

Kelsey, J. E. 1009

Kendall, C. E. 1901

Kendall, R. F. 0937

Keogh, P J 1988

Kerkhoffs, P. L. 2057

$\begin{array}{ll}\text { Kern, W. } 0903 & 1882 \\ \text { Kernahan, J. A. } 0052 \text { Kolomijcuk, V. } 0209\end{array}$

Kerr, J. A. 1095 Kolosjuk, V N. 0392

t. Kesselman, V. S. 0359 Kolosov, M. N. 1287

Kaempf, B. 1272,1273 Kessenich, A. V. 0095
Kagan, J. M. $0140 \quad$ Kettle, S. F. A. 0023

Kettrup, A. 1645

Khan, M. A. S. 1567

Khalil, O. S. 1040

Khubchandani, P. G. 0015

Kibbel, H. U. 0842,

0843, 0844, 0845

Kali-Chemie AG 1826 Kicska, P. A. 1063

Kalinin, G. P 0309 Kiefer, B. 1903

Kalis, T. V. 0443 $\quad$ Kifer, K. 0049

Kaljuznaja, V. G. 0063 Kikuchi, S. 1041

1940,1941 W. 1927

Katzschmann, E. 2028 Kneubuehl, F. K.

$\begin{array}{lc}\text { Kauer, J. C. } 1221 & 0387 \\ \text { Kawahara, F K. } 1957 \text { Knorozova, G. V. } 132\end{array}$ nubovec, R. G. 0874 1664

obayashi, T. 170

Kocergin. I. 0653

Kocergina, L. A. 0549

Koch, A. L. L. OTh1

Kocher, J. 0618

Koeppendoerfer,

Koga, Y. ${ }^{1699}$

Kohlhaas, E. 1768

Koibuchi, M. 1331

Kojro, Z. 0930

Kolb, E. D. 1868

Kolbe, A. 0936

Kolesnikova, B. M.

Kolitz, B. L. 158

Kolling, G. 1349

lobichin,

$\begin{array}{lll}\text { Kolomijcuk, V. } & 0209 \\ \text { Kolosjuk, V N. } & 0392 \\ 0\end{array}$

Komissarova, L. N.

0621

Komnik, J. F. 0154

Kondo, T. 0928

Kondow, T. 0081

Konjuchov, V. K. 00

Konjuchova, N. B.

Konovalova, M. V.

078

Kilin, V. S. 0802

Kim, Pu-Jun 1188

Kimball, C. W. 0518

King, H. W. 0270

Kiprianov, G I. 13

1105

Konstantinov, J. S. 0093

Kopcik, V A. 0506

Kopylov T. 0036

Korableva, A A 078

Koreeda, A. 0306

Korenjako, A. I. 1286

Koriyama, $Y .1440$

Korobejnicev, O.P.

0797
Korolev, V. E. 1591



Kirimura, J. 1921

Kirkbright, G. F. 1581 Korolkov, N. S. 1597

Kirjuchin, N. N. 0510 Korovin, N V. 0687

Kirjuchin, V. K. 1313

Kiseleva, K. . $\mathbf{0 1 5 1}$

Kishi, T, 1355

A. 1287 Korsak, V. V. 1783

Kislovskij, L. D. 0379,

Kiso, $Y$ 1643, 166

Kispert, L. D. 1001 1273

Kosenko, L. V. 1290

Kosevic, v. M. 0185

Kost, L. L. 0602

Kosuchin, V. V. 036?

Kotval, P. S. 0670

$\begin{array}{lll}\text { Koutecky, J. } & 1016 \\ \text { Kovacs, C. A. } 1214\end{array}$

Kovacs, C. A. 1214
Koval, A. G. 0036

Kitaoka, Y. 1643,

1064 P. 1081

Kladkevyc, M. D. 049

Klassen, D. M. 0043

Klatt, L. N. 1575

Klayman, D. L. 1703

Klein, E. 2058

Klejner, E. M. 128
Klesnil, M. 0323

Klimenko, N. M. 0010

Klimov, V. V. 0487

Klimovic, A. T. 181

Kljagina, A. P. 0010

Klocman, S. M.

Kosa, J 1466

E. 1708

Koziara, A. 0853

Kozin, L. F. 0664

Kozlowska, H. 0930

Kracino, T. V. 044

Kraft, G. 1574

Kralina, A. A. 0163

Krasnikova, A. J.

0506

Krasnovskij, A. A.

Krauss, A, 1706

Kravcov, C. I. 0692

Krebs, B. 0258, 0865
Kreiskott, H. 1485

Krekeler, H. 2019

Krem
Keberle, H. 1454

Knorring, O. v. 0879 Kremencuhskyj, L. S, Lachance, G. R. 0881,

Rocina, M. P. 1550

Kodama, Y. 1079

Koehler, A. M. 1596

Kolotilova, V. J. 0596
Komeno, T. 1506, 1508

Kommandeur, J. 1059

Konarski, J M. 1022

0011
Konorov, P. P. 0694

Konovalov, L. V. 0077

Konyseva, N. M. 1675

, Korzenjak, I. G. 0586

Klockov, $V$, 0033

Kovalenko, E. S. 0432 ,

Kovalenko, V. V. 0193

Kray, L. R. 1205

0496 . L. S. Lacha 0882

Krespan, C. G. 1209

Krestov, G. A. 161

Krestovnikov, A. N

Kreutzmann, J. 0778

rewel-Leuffen

GmbH 1404

Kricker, W. A. 1933

Laider, K. J. I126,

Krieghoff, i G. 0278 Lalancette, R. A. 0229

Kriese, R. 0972 Lam, D. J. 0528

Krimm, S. $0919 \quad$ Lamb, D. 1790

Krishnan, M. 1155 Lamberton, J. A. 1055

Krishnan, V. R. 1710 Lambourne, R. 0757 
Lieu Van, T. 1726 Ligny, C. L. de 0685 Lilga, K. T. 100 Lilly, E. \& Co. 1518 Lim, E. C. 0925, 092 Lima, F. W. 167

Lin, M. C. 1126, 1130 Lin, Tan-Wan 116

Lin, W. S. 1048

Lindberg, G. W. 118

Linden, J. G. M. Van der 1662

Lindenmann, A. J.

1465

Lindner, E. 1444, 1453

Linggood, F. V. 1526

Link, J. K. 0119

Liphia Lyonnais

Pharmaceutique

Lipkin, F. M. 1589

ipscomb, W. N.

Liptay, W. 0915

Lisovskij, F. ${ }^{0491}$

Littler, J. G. F. 1143

Litvin, J. A. 0644 1638

Litvinenko, V. I. 0603 1304

Liu, Kang-Jen 1266

Livage, J. 0290

Livingston, K. 0072

Ljubcenko, A. V. 041

Ljubutina, L. G. 0

Lobacev, V. P. 0465

Lobas, L. M. 0608

Lockhead Aircraft Corp. 184

Loeffler, W. 1524

Loepfe, E. 1609

Loev, B. 1448

Loev, L. 1448

Lord, G. H. 1384

Lorenz, C. E. 200

Lorenz, D. 1350

Lorenzelli, V. 0938

Lorenzo, S. De 1213

Losev, S. A. 0037

Lovcikov, V.S. 18

Fabrik Produk

tions aktieselskab

1513

Low, M. J. D. 0753

Lown, J. W. 1236 ,

ubkowitz, J. A. 0910

Lubyanov, L. P. 1546 Malhotra, S. K. 1146

Lueiv, 0482 Luettringhaus, $A$ 2047, 1042

Malinina, I. G. 0322
Malinovskaja, G. F. 0537

Lugenheim, w. 1986
Lugovoj, v. N. 0375

Lugt, W. van d

Lukas, P. 0323

Lukasenko, E. E. 0561

Luke, C. I. 1648.

1774
Luk'janyceva, V. I.

ukoveev, P. D. 070

Lukovcev, P. D. 0708

Lutfulin, K. T. 130

Lutz, A. H. 1472

Lutz, A. W. 1213 ,

Lutz, R. E. 1202

Luznaja, N. P. 0637

Lvovic, B. I. 0616

Lynch, C. T. 1856

Lyness, W. J. 1902

Lyscov, A. I. 0180

M. \& T. Chemicals, Inc. 198

Má, J. C. N. 1236, 1237 McArthur, K. 1769 Malinovskaja, G. N 1288

Malinowski, E. R.

0959 Galjukov, B. A..

Maljukov, B. A. .1

Malli, M. 0206

Malnjev, A. F. 0496

Malrieu, J. P. 1013,

Maltese, M. 0070

Malvano, R. 1607
Malysev, V. N. 0613

Mamedaliev, F. D.

0486

Mamontov, E. A. 0343,

1819 a 0177

Merca, E. 0068

Manasevit, H. M. 0165 Merck, E. AG 1467

Manchester, K. E. 0361 Merck \& Co., Inc:

Mandelbaum, A. 1007

Mankinen, $\mathrm{L}$. 1265

Mann, R. S. 1778

Manna, S. 1339

Mannan.

K. A. I. F. M. 0256

Manning, P. G. 0872,

0873 .

Manufacture de

ceutiques A Christiaens Soc. An.
$\mathbf{1 5 1 4}$

McCarron, E. A. 1803 Mapleton, R. A. 0126

McChesney H. R. 0038 Maraev, S. E 0307

Macchia, J. T. La Marcou. A 0935

0376

Marcoux, L. S. 0994

McClusky, F. K. 1068 Mard
McConkey, J. W. 0052

Marsak, E. M. 0777

Marshall. T. 0117 Matsen, F. A. 0014,

Mendez, V. 1

Meng, K. 1449

Michajlicenko,
Marfunin, A. S. 0519 argrave, J. L. 0107 Maria, H. J. 0924 Marinier, B. 116 Marquis, D. M. 1938 Marr, C. 1344 Marshall, J. A. 1181, 119 Martel, J. 1950 Martin, F. M. 1497 Martin, J. W. 0317 Martinek, C. $\mathbf{0 3 3 5}$ Masarnau, J. de D. 1890

18sarov, S. I. 0519

Maslennikova, V.J. 0579

Masiov, P. G. 0548

Mason, B. J. 0560

Massart, D. L. 1672

Massat, A. 0941

Masumjan, V. J. 12 Masuzawa

Mateescu, G. D. 1116 Matejicek, A. 1883 Matheson, A. J. 1061 Mathieu, J.-C. 0554 Mathur, S. C. 0533 Matrosov, I. V. 0031 0017

Matsumoto, T. 1640 0120

atsuo, T. 0955, 0956,
1076, 1077

Matthijsen, A.P.

Mattoon, W. 0972

Matwiyoff, N. A. 0102

Mauli, R. 1524
May jun., J. A. 1274

Maynard, M. M. 1241 Mayrhofer, O. L. ${ }^{1}$ Mazdiyasni, K. $\mathbf{S}$.

185

Mazurenko, N. D.

Mecke, R. 1042

Medeck, E. 1588

Mednis, P. M. 0454

Mee, C. H. B. 0418

Meguro, T. 1402

Mehra, A. 0044 1059

Mehrotra, N. K. 0945

Mehrotra, R. C. 1108
Mehta, N. B. 1348

Meier, $T$. 0663

Meijer, j. W. A. 1621

Mejsner, L. B. 0502

Meldrum, P.R. 163

Melnikova, L. V. 0345

Mendelsohn, N. 183

Montecatini Edison

1456, 1461, 1462, 15

$1511,1910,1922$
Merlo, L. 1866

Merlo, L. 1866
Merrick, H. F. 0321

Merzanov, A. G. 0772

Meth-Cohn, O. 1083

Mets, E. 0350

Metzger, H. 2037

Metzler, G. 1975

Meyer, K.-H. 1512

Meyers, E. 1436

Meystre, C. 1510

Micev, I. 1736

0536

Michajljuk, G. P. 0482

Michajljuk, G. P. 0482
Michajlov, S. K. 0312

0731
Michajlov, V. V. 0151 Morgenstern, K. 203 Michajlova, N. A. 0504 Morgunova, A. S. Majlova, S. S. 0756061

Michajlova, T. V. 1323 Moriarty, R. M. 1100 Michel, C. 0238 290 Morishima, I. 0958 Michener, A. W. 1872 Morishita, M. ${ }^{1260}$ Middleton, W. J. 1209, Moritani, T. 1420 1217 Moritz, A. G. 098 Miguler, A. 1859 Morrison, G. C. 122 1671 A. Z. Morrs

Miles, M. G. 1147

Miles Italiana M. I. D. A. S. p. A.

Mileson, J. S. 0217

Millar, I. T. 1192

Miller, I. R. 0730 .

Miller, R. J. 1544

Minami, S. 1440

Minamikawa, $\mathbf{T}$. 1331

Minasso, B. $104 \overline{5}$

Minnis, N. 0491

Minister of Technology 1935

Mironov, V. E. 0077

Misdolea, c. 0546

0366

Mishra, M. B. 0044

Miskei, M. 1605

Mislavska, r. S. 1280

Misiuk, A. 0529,0530

Mitooka, M. 1640

Miyai, $\mathbf{K}, \mathbf{M}$.

Miyaji, N. 1691

Miyajima, K. 0932

Miyaki, M. 1259

Miyatake, O. 0110

Miyazaki, M. 1257

Mizushima, $S$. 0403

Mkrtcjan, A. R. 0519

Moakley, D. F. 1146

Mocalov, $\mathbf{K}$. N. 0779

Mochimaru, F. 0700

Modrow, $H_{\text {; }}-W .2067$
Moedritzer, K. 2025

Moehler, K. 1704

Moehrle, H. 1312

Moeken, H. H. P. 175

Moffatt, J. G. 1250

Moffett jun., L. R.

Moffett, R. B. 1483

Mohaupt, G. 0351

Mokeeva, R. N. 1713 1805

Mole, M. F. 1929

Moleva, N. I. 1319

Molnar, F. 2021.

Molnes, A. G. 0457

Molotílov, B. V. 0321

Mondet, S. 0246

Monkman, J. L. 1541

Monnier, D. ${ }^{1609}$ 1490

Monsanto Co. 1854

Montgomery, H. A. C.

Montecatini Soc. G

per l'Industria

Chimica 1866

Moody, J. R. 1700

Mookherji, A. 053

Mooney, R. W. 0390

Moore, J. E. 2010

Moore, J. W. 0212

Moore, P. B. 0279

Moracevskij, 0998 0660

Morand, M. 1548

Moreaul, 1548

Morel, J. 1531

Moret-Bailly, J. 0918

Torfopoulos,
V. C. P. 0695

ritz, F. L. 0165

Morton, A. H. 0364

1876

Moser, J.-F. 0387

Mosidze, V. P. 0822 


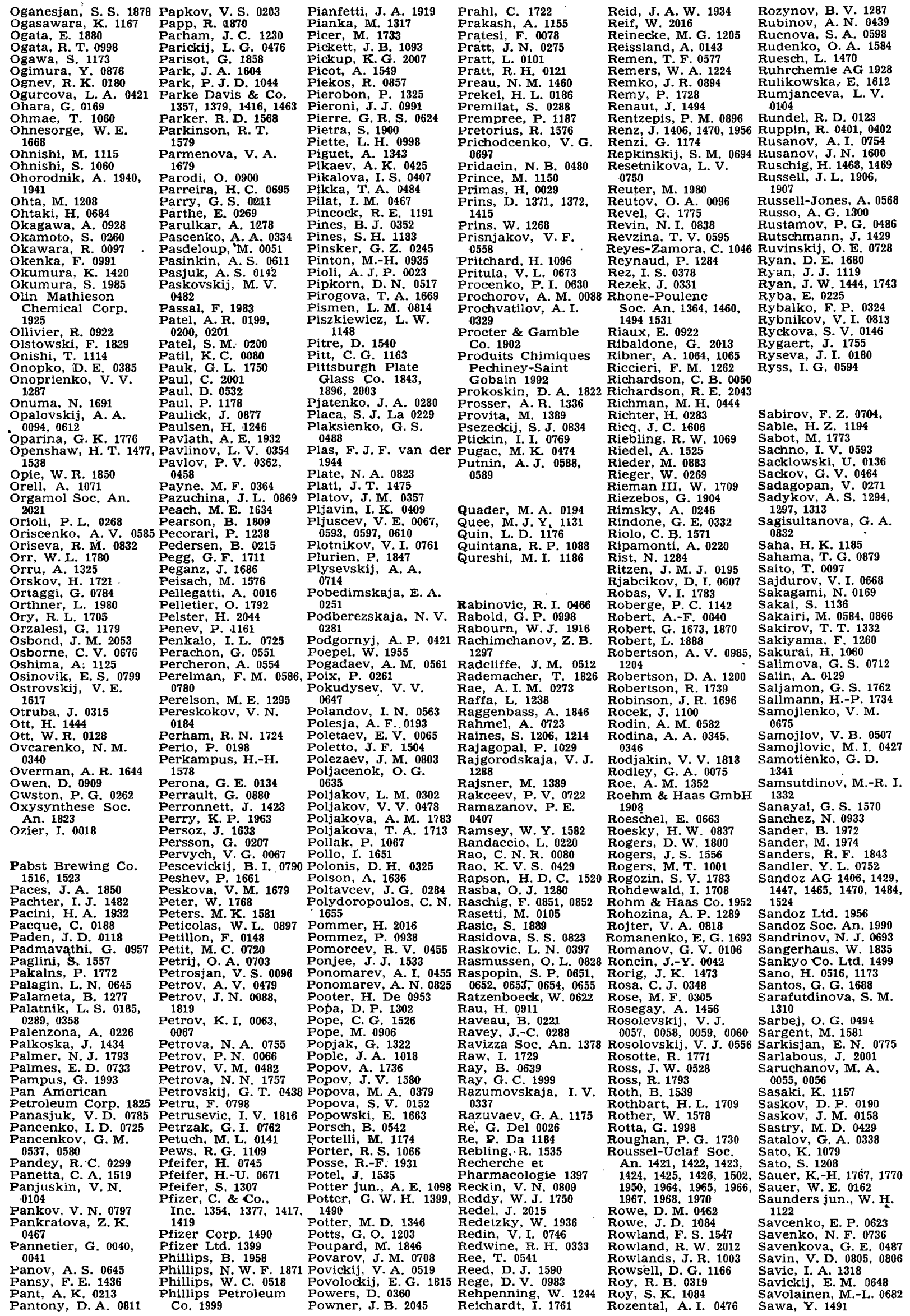




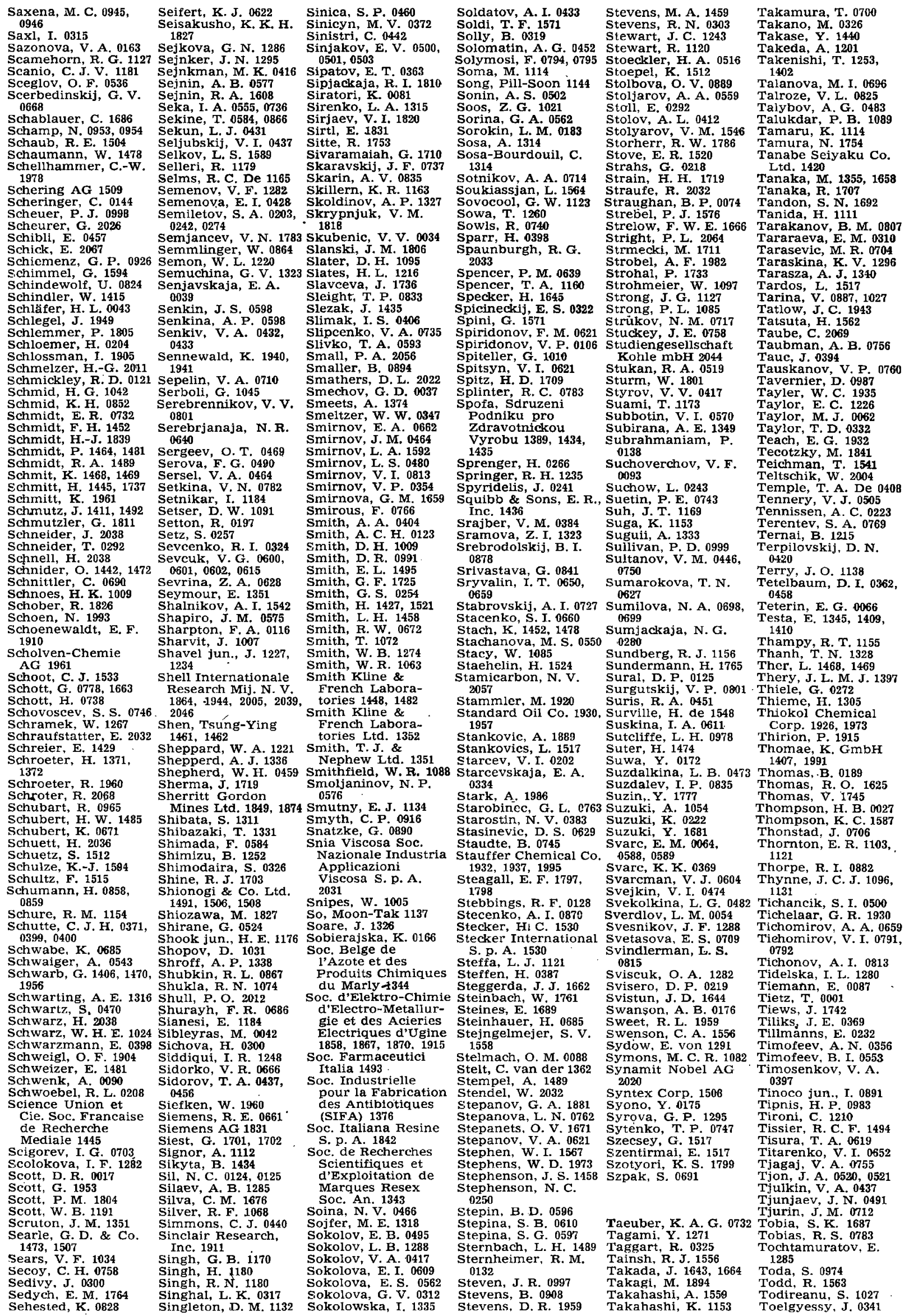


Tolmacev, V. N. 0765 Union Oil Co. of Tolstaja, S. N. 0756 Californla 1933 Tom, w. 1211 United States of $\begin{array}{ll}\text { Toma, S. Z. } 0390 & \text { America Secretary } \\ \text { Tomalia, D. A. 1199 } & \text { of Air Force 1856 }\end{array}$

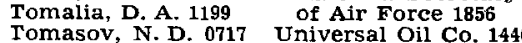
Tomasov, N. D. 0717 Tomilin, I. A. 1820 Tomilov, A. P. 0693 Tomilov,

Pangarova, T. 1337 Universal Oil Products Co. 2027 Products Co. 2027
Uno, T. 0931, 0932, Uno,

0943 pjohn Co.

Tompa, K. 1545 1483,1942



Toren jun., E. C. 1565 Urjaev, I. A. 1550
Tori, K. 1111 Usakev. $\mathrm{J}, 0600$

Toriyama, K. 1004, Uvarov, A. V. 0756

Toropov, B. A 0488

Torrálba, M. 0308

Tourres, D. A. 1695 vagh, A. S. 0201

Tovbin, M. V. 0821 Vagina, N. S. 0607

Townsend, J.R. 0147 Vaira,. M. Di 0268

Toth, F. 1545. 165

Toth, F. 1545

Toyama Kagaku

Toyo Koatsu

1898

Valdre, U. 1601

Valjasko, E. G. 0397

Vancamp, R. M. 2003

Vance, R. L: 0839

Vandenbroukke, $\mathbf{w}$,

0954. 0988

Vanderheiden, B. S

1718

Trahanovsky, w. S.

1110

Vantorex L.td. 1400

Varfolomeev, M. B.

Tranchant, J. 1652

Trautwein, N. L. 16

Trebillon, E. 1992

Treinin, A. 0830 006 ?

Varga, F. 1517

Varga, S. 0341

Varina, T. M. 0388

Trepanier, D. L. 1488 Vascenko, V. I. 0392

Treskuriova, R. L.

0755

Vasileiskaja, N. S. 1175

Tretjakov, J.P. 0142 Vasilescu, A. 1116

Tribunescu, P. 0068 Vasilev, V. P. 0549 Tric, C. 0900
Trimofeev, V. B. 0392 Vasjutinskij, N. A.

Trofímov, G. V. 0605 ,

rrofim

Trombe, F. 0554

Troponwerke Vaskivnjuk, V.I. 1282

Vasko, A. 028 Dinklage \& Co. 1350 Vasu, K. I. 0811
Trusov, G. N. 0721

Trusoviatows Gi, w.

0529,0530 0494

Vatolin, N. A. 0650

Trzebowski, N. 0944 Vavilov, V: S. 0452

Tsujimura, S. 1559 VEB Deutsches

Tsuruo, I. 2024

Tuedoes, F. 076

Tull, R. J. 1456

Tullock, C. W. 1899

Tunickij, L. N. 0035
Tunickij, L. N. 0035

Turcinova, L. N. 0734

Turjan, J. I. o728

Turnb2 Hydrierwerk Rodleben 2030
VEB Farbenfabrik Wolfen 2067

Vechter, B. G. 0405

Veening, H. 1642

$\begin{array}{ll}\text { Veening, H. 1648 } & \text { Waal, M. J. } 1944 \\ \text { Vejeman, A. M. } 1319 & \text { Wachter, R. De } 162\end{array}$

Velickov, A. M. 13 .

Velikanova, L. V. 0591

Turner, D. J. 1070

Veltman, H. 1874

Venanzi, L. M. 0045

Venerovskaja, L. N

Venkatesan, N, 0983

Uchanov, J. I. 0471

Uetani, K. 0876

Vercellot, $\mathrm{V}$. N. R. 173 Vercner, N. 1600

Ukrainskij, J. M. 1591

Uliman, R. 1266

Verescagin, L. F. 0152 2, Wainerdi, R. E. 1688 Verma, G. S. P. 108

Ulrich, $H .1931$ Vermeil, C. 1142

Umebayashi, H. 0524 Vernova, T. P. 1881

$\begin{array}{ll}\text { Unilever- } & \text { Veselago, L. S. } \mathbf{1 6 6 9} \\ \text { Emery, N. V. } 1917 & \text { Veselov, M. G. } \mathbf{0 0 0 8}\end{array}$

Veselovskij,
Vetri, G. 0382

Vijjan, V. K. 1170

Vladimiroff $T$.

Oladimirov, $G$. G.

Vorobev, G. A. 040

Voronip, J. M. 1600
Viaene, W. 0642

Walters, D. B. 1800 Vidjakina, L. V. 0614

Viktorov, I. A.

Vilenskaja, B. G. 0800

Vilk, J. N. 0445, 0583,

Vinogradov, A.V.

inogradova, G. Z.

Vinokurova, E. B.

0820

Viout, A: 1918

Vizsolyi, A. J. 1874

Vjazankin. N. S. 009

Vjazmenskij, J. E.

jugina, A. F. 0789

lasenko, K. K. 0550

Vlismas, I. V. 061

Vo, Kim-Phan 1143

od7

Voellenkle, H. 0235 , 0236

vojeechovskaja, $\mathbf{O} . \mathbf{K}$ 0032

Vogel, E. 0965

Vogel, G. 1468, 1469

Vogler, $\mathrm{K}$. 1534

Vogt, W. 1940, 1941

Volckov, A. K. 1812

Vold, R. L. 0968

Voliotis, S. D. 1655

Voljak, L. D. 0567

Volkov, A. M. 0719

Volkovic, A. V. 0652

Volman, D. H. 1141

Volodko, V. G. 0362

1320

Vondracek, M. 143

Voronin, J. M. 028

Voronkev, A. A. 02
Vulich, A. I. 0748

Wadsworth jun.,

W.S. 123

Waeppling, R. 0319

Waggoner, A.S. 099

Wagner, A. 1978

Wagner, C. D. 112

Wagner, J. 1760

Wagner, $k$ : 1960

Wagner, R. S. 0156

Wainai T 1681

Waite, R. O. 1227

Wald, F. 0641

Waldbaum, D. $R$.

0571

Walkenstein, S. S. 160

Walker, F. H. 1932 1492

Wang, C. S. 0733

Ward, J. C. 0997

Wares, G.W. 0117

Warkentin, J. 113

Warneck, P. 0131

Warner, J. 1602

Warner-Lambert

Co. 1428

Warren, M. R. 0311

Warren, S. W. 1583

Wassermann, G. 030

Wassermann, G. ${ }^{03}$

Watanabe, $\mathbf{K} .012$

Watanabe, S. 1153
Watson, P. G. 0508

Watson, P. G. 0508

Watson, R. W. 19

Watts, R.

Wawzonek, S. 1241

Weatherburn, D. C.

Webb, T. G. 0127

Weber, H. 1451



Weber, T. A. 1944

Webster, B. R. 1011

Webster, S. J. 0831

Weeks, C. E. 179

Weidelt, J. 0228

Weidenberg, H. 0915

Weigmann, H.-J. 09
Weik, R. W, 1795

Weik, R. W. 179

Weil, J. A. 1051 .

Weingarten, H. 1147,

Weinstock, J. 1482

Weinstock, $\dot{\mathrm{L}}$. T. 1235

Weir, J. 1876

Weis, G. 1784

Weisenborn, F. L. 1436

Weiss, M. J. 1224, 1504

Weissert, J. 2063

Welleome Foundation

Ltd. 1348, 1477, 1526,

1529. 1538, 1539

Wells, C. H.J. 0940

Wells, N. 1620

Wendisch, D. 0981, 1047

Wendler, N. L. 1183

Wendling, E. 008

Wenner, W. 1475

Wentworth, W.

West, R. 0847, 0848,

0849,0850

West, T. S. 1581, 1587

Western Electric Co.,

Inc. 1868

stinghouse

Electric Corp. 1869

Westrum jum

Wetroff, G. 1992

Wettstein, A. 1510

Weyer, R. 1451

Wharmby, M. 1232

Whiffen, D. H: 096

White, A. D. 0391

White, J. B. 2027

White, J.R. 1935 Whittacker, N. 1477,

1538

Whittaker, B. 1561

Whitton, J. S. 1620

Wickersheim, K. A.

1841

Wieland, P. 1510

Wiersma, D. A. 1059

Wiest, H. K. 1913

Wijn, H. P. J. 1678

Wilhelm, M. 1454, 1
Wilkens, M. 0191

Wilkens, M. 0191

Wilkins, C. J. 0075

Will, G. 0525

Williams, A. W. 2018

Williams, D. J. 0770

Williams, $\mathrm{D}$. $T$. 1585

Wilman, H. 0327

Wilson, R. K. 0923

Winegard, W. C. 0157

Winer, A. M. 0963

Winkler, H. 1406

Wirth, H. O. 0893,

Wirth, M. M. 2000

Wirth, W. 1485

Wiseman, H. G. 175

Witkowska, B. 1651

Witte, J. 1993

Wittmann, A. 0235 , 